\title{
Conocimientos y prácticas sobre lepidopterismo por Hylesia metabus (Cramer, 1775) (Lepidoptera: Saturniidae) en la parroquia Yaguaraparo, Estado Sucre, noreste de Venezuela
}

\author{
Chloé Herrera-Chaumont ${ }^{1}$, Mayira Sojo-Milano ${ }^{2,3}$, Luis Pérez-Ybarra ${ }^{4}$ \\ ${ }^{1}$ Departamento de Epidemiología del Municipio Sanitario Mariño, Fundasalud, Irapa, Estado Sucre, Venezuela. ${ }^{2}$ Dirección \\ de Control de Vectores, Reservorios y Fauna Nociva, Dirección General de Salud Ambiental, Ministerio de Salud, Maracay, \\ Estado Aragua, Venezuela. ${ }^{3}$ FUNINVEST, Fundación Venezolana para la Investigación Multidisciplinaria, Maracay, Estado \\ Aragua, Venezuela. ${ }^{4}$ Escuela de Bioanálisis Sede Aragua, Facultad de Ciencias de la Salud, Universidad de Carabobo, \\ Maracay, Estado Aragua, Venezuela.
}

\section{RESUMEN}

Introducción. El lepidopterismo es definido como el conjunto de afecciones causadas por el contacto del ser humano con diversos géneros y especies del orden Lepidoptera. La hembra de esta especie de mariposa presenta en su abdomen pelos o setas que contienen toxinas las cuales al entrar en contacto con la piel y mucosas generan dermatitis y lesiones de la mucosa ocular, orofaringe y tracto respiratorio superior, por lo que es necesario desarrollar mejores estrategias educativas para minimizar el impacto sobre la salud.

Objetivo. Se realizó la caracterización de los conocimientos y prácticas locales para la prevención y control del lepidopterismo por Hylesia metabus en la parroquia Yaguaraparo, noreste de Venezuela.

Materiales y Métodos. 400 personas fueron seleccionadas al azar y encuestadas, las respuestas fueron categorizadas mediante una escala de 0 a 4 puntos (4 para el mejor conocimiento/ práctica), las dimensiones exploradas fueron: conocimientos sobre H. metabus, lepidopterismo, prevención y control de lepidopterismo, y conocimiento y prácticas en general. Se aplicaron estadísticos descriptivos, ANOVA y la prueba de comparaciones múltiples de Tukey, considerando sexo, grupo etario, educación y ocupación como variables intervinientes.

Resultados. El conocimiento sobre H. metabus fue bueno $(\bar{x}=2,61)$; sobre lepidopterismo fue regular $(\overline{\mathrm{x}}=1,65)$, las prácticas de prevención fueron buenas ( $\bar{x}=2,52)$, así como las de control $(\bar{x}=2,74) .97 \%$ declararon haber padecido lepidopterismo; $71 \%$ usan tratamiento médico y casero, el $29 \%$ restante usa tratamiento casero y automedicación. La medida más conocida y utilizada para evitar el contacto con la mariposa fue apagar las luces $(50,2 \%)$. La aplicación de insecticidas fue la medida más frecuente considerada útil para eliminar las mariposas del ambiente $(68,2 \%)$. Ante el prurito las acciones incluyeron tratamiento con antihistamínicos $(30,7 \%)$ y casero $(27,4 \%)$, siendo la aplicación de alcohol la más utilizada $(23,5 \%)$.

Conclusiones. La educación y ocupación mostraron influencia significativa en las respuestas. La población de la parroquia mostró nociones buenas, aunque no óptimas, sobre lepidopterismo y sus prácticas requieren mejorar, para obtener en conjunto un mejor nivel de

Autor para correspondencia: Luis Pérez-Ybarra, Final Avenida Ruíz Pineda, Universidad de Carabobo, Facultad de Ciencias de la Salud, Sede Aragua, Escuela de Bioanálisis, Departamento de Ciencias Básicas, Sector La Morita, Santa Rita, Municipio Francisco Linares Alcántara, Estado Aragua, Venezuela, Zona Postal: 2103. E-mail: Lmpy2005@gmail.com

Recibido: el 26 de junio de 2015 Aceptado para publicación: el 9 de octubre de 2015

Este documento está disponible en http://www.revbiomed.uady.mx/pdf/rb162713.pdf 


\section{Herrera-Chaumont et al}

potencial de participación ante el problema.

Palabras clave: Hylesia metabus, conocimientos, prácticas, lepidopterismo

\begin{abstract}
Knowledge and practices about lepidopterism by Hylesia metabus (Cramer, 1775) (Lepidoptera: Saturniidae) in Yaguaraparo parish, sucre State, northeastern Venezuela

Introduction. Lepidopterism is defined as the set of conditions caused by human contact with various genera and species of the order Lepidoptera. The female of this species of butterfly posses hairs that contain toxins which upon contact with skin and mucous generate dermatitis and lessions in the ocular mucosa, oropharynx and upper respiratory tract therefore, it is necessary to develop better teaching strategies to minimize the impact on health.

Objective. Characterization of local knowledge and practices for the prevention and control of lepidopterism by Hylesia metabus in the parish Yaguaraparo, northeastern Venezuela, was carried out.
\end{abstract}

Materials and Methods. 400 people were randomly selected and surveyed, responses were categorized using a scale of $0-4$ points ( 4 for better knowledge/practice), the surveyed dimensions were: knowledge about H. metabus, lepidopterism, prevention and control of lepidopterism, and knowledge and practices as a whole. Descriptive statistics were applied, as well as ANOVA and Tukey test for multiple comparisons, considering sex, age group, education and occupation as intervening variables. Knowledge about $H$. metabus was good $(\overline{\mathrm{x}}=2.61)$; on lepidopterism was moderate $(\overline{\mathrm{x}}=1.65)$, prevention practices were $\operatorname{good}(\bar{x}=2.52)$, also for control $(\bar{x}=2.74) .97 \%$ declared lepidopterism experience; $71 \%$ used medical and home treatment and the remaining 29\% used home and self-medication treatment. The better-known and used measure to avoid contact with the butterfly was turning off lights $(50.2 \%)$. Insecticide application was the most frequent measure considered useful to remove butterflies from environment (68.2\%). Against itching, actions included taking antihistaminic (30.7\%) and home treatment $(27.4 \%)$, being topic alcohol the most used (23.5\%). Education and occupation had significant influence on given responses.

Conclusion. Parish population showed good notions on lepidopterism, though not optimal, and their practices require improvement, in order to combine for a higher participation level to face the problem.

Key words: Hylesia metabus, knowledge, practices, lepidopterism

\section{INTRODUCCION}

El lepidopterismo se define como el conjunto de afecciones causadas por el contacto con diversos géneros y especies del orden Lepidoptera. Se refiere a efectos adversos por contacto con polillas y mariposas y se manifiesta como dermatitis, urticaria y reacciones sistémicas (1-3). En América, principalmente en Costa Rica, México, Trinidad, Guyana Francesa, Surinam, Brasil, Uruguay, Venezuela, Perú y Argentina el lepidopterismo ha sido atribuido al género Hylesia (4).

En Venezuela, esta entidad clínica se asocia específicamente con el contacto directo o indirecto con Hylesia metabus (Cramer 1775) (4-6). La hembra de esta especie de polilla, en general llamada mariposa, por su ubicación taxonómica como lepidóptera, presenta pelos en su abdomen, que usa para la protección y cobertura de su postura $(7,8)$; estos pelos también son denominados setas, dardos, espículas o flechettes, $(4,8)$ y contienen toxinas erúcicas que al entrar en contacto con la piel y mucosas generan dermatitis y, en menor proporción, lesiones de la mucosa ocular, orofaríngea y del

\section{Revista Biomédica}


Lepidopterismo por $H$. metabus en Yaguaraparo

tracto respiratorio superior $(4,9)$. En la zona nororiental de Venezuela, (estados Sucre, Delta Amacuro y Monagas) representa un importante problema de salud pública, ya que genera un alto impacto ambiental, forestal, social y económico $(7,10)$.

La parroquia Yaguaraparo del municipio Cajigal en el estado Sucre, ha sido afectada por las apariciones de H. metabus desde 1952 (11). Las primeras crónicas en Venezuela provenientes de esta parroquia, relatan su existencia cómo se acercaba al alumbrado público y cómo las personas que se encontraban cerca, sufrían lesiones (5). Desde esa época y en la actualidad, cuando ocurren las apariciones de H. metabus, localmente llamada mariposa y "palometa peluda" (6), las actividades cotidianas de la zona se ven alteradas debido a la afectación de los pobladores o por el temor de ser afectados, su presencia obliga a mantener las casas y establecimientos cerrados y a oscuras para no atraer al insecto (11) que siente predilección por la luz, especialmente, por luz halógena; las hembras de esta especie, al revolotear alrededor de los focos de luz desprenden las setas o pelos urticantes, que al ser dispersados por el viento y entrar en contacto con las personas producen dermatitis y otras manifestaciones $(5,7)$.

Esto genera un fuerte impacto sanitario, ya que durante las apariciones de H. metabus, se existe una concentración de recursos económicos y humanos en las áreas endémicas, a fin de atender los numerosos casos de lepidopterismo que se presentan (12). Adicionalmente, el insecto causa impacto ambiental, por la defoliación que ocasiona al alimentarse de las hojas de las especies del bosque manglar, fundamental para el ecosistema marino (12) como el mangle rojo, (Rhizophora mangle), el mangle negro (Avicennia germinans) y el mangle blanco (Langucularia racemosa) $(7,11)$. Estas comunidades y hábitats comprenden las áreas de trabajo que enfoca el Programa Nacional de Vigilancia y Control de $H$. metabus, estructurado por el Ministerio de Salud para reducir el alcance de este problema de salud pública en Venezuela.

Durante las apariciones de H. metabus, los habitantes de la parroquia Yaguaraparo han incorporado a su cultura un conjunto de conocimientos y prácticas tradicionales para prevenir y controlar los efectos indeseables del lepidopterismo. Se trata de sistemas que requieren ser documentados y sistematizados, dada la repercusión del evento a nivel local y hacia áreas vecinas. En tal sentido, fue objetivo de este trabajo caracterizar los conocimientos y prácticas relacionados con esta entidad en la parroquia, con el propósito de aportar a la comprensión de su dinámica y obtener la información necesaria para contribuir al desarrollo de una comunicación efectiva en salud, facilitar la transferencia de los resultados de la investigación, fortalecer las políticas de promoción de la salud en el contexto y favorecer la seguridad humana en salud, al reducir el impacto indeseado en la calidad de vida de los habitantes de estas áreas.

\section{MATERIALES Y MÉTODOS}

La investigación se realizó durante el año 2010 en la parroquia Yaguaraparo del municipio Cajigal del estado Sucre, Venezuela, ubicada a unos $580 \mathrm{Km}$ al este de Caracas, $10^{\circ} 34^{\prime} 11^{\prime \prime} \mathrm{N}$ y $62^{\circ} 49^{\prime} 45^{\prime} \mathrm{W}(13)$. Esta parroquia tiene una extensión de $116 \mathrm{Km}^{2}$, con una población aproximada de 11.030 habitantes (14), la elevación se encuentra entre 0 y $200 \mathrm{msnm}$, la temperatura oscila entre $25-27^{\circ} \mathrm{C}$ promedio anual y las precipitaciones entre 1000 y 1200 mm anuales (15). Sus comunidades son rurales, donde predominan la agricultura y la pesca como actividades económicas. La cercanía de los bosques manglares, pertenecientes a una intrincada serie de caños que forman parte del Golfo de Paria, hace a la parroquia vulnerable al lepidopterismo, por ser estos bosques el hábitat natural de $H$. metabus $(7,11)$. 


\section{Herrera-Chaumont et al}

Se llevó a cabo un trabajo de campo, observacional, descriptivo y transversal, basado en una encuesta estructurada validada por expertos y de la cual se hizo una aplicación piloto antes de iniciar el trabajo con la muestra del estudio. La validación de expertos fue realizada por dos sociólogos y un malariólogo especializado en $H$. metabus; una vez incorporadas sus observaciones, se hizo el piloto de la encuesta en el campo en la comunidad de Río Seco de Venturini, capital de la parroquia Libertad. Esta es una parroquia vecina y geográficamente similar a Yaguaraparo, con antecedentes como zona afectada por la aparición de H. metabus. Allí se aplicaron, al azar, 40 encuestas a lo largo de la zona, ensayando los criterios de inclusión/exclusión, la verbalización y el tiempo de administración del instrumento. A partir del análisis y discusión de los resultados de esta prueba piloto se modificaron algunas preguntas de la encuenta final, en cuanto a redacción, parafraseo y alternativas de respuesta. La encuesta estructurada incluyó cinco dimensiones para el análisis, a saber: 1) conocimientos sobre $H$. metabus, 2) conocimientos sobre lepidopterismo, 3) prácticas respecto a la prevención del lepidopterismo, 4) prácticas respecto al control del lepidopterismo, y 5) conocimientos y prácticas en general (es decir, la integración de los aspectos explorados, tanto para conocimientos como para prácticas). Las

\section{Cuadro 1 \\ Escala de puntuación y categorías de las respuestas de la encuesta estructurada}

\begin{tabular}{cc}
\hline Puntuación & Conocimiento/Práctica \\
\hline 0 & $\begin{array}{c}\text { No conoce,desconoce/No realiza prácticas } \\
\text { Escaso conocimiento/Prácticas poco } \\
\text { favorables }\end{array}$ \\
2 & $\begin{array}{c}\text { Conocimiento regular/Algunas prácticas } \\
\text { favorables }\end{array}$ \\
3 & Conocimiento bueno/Buenas prácticas \\
4 & Conocimiento óptimo/Prácticas favorables \\
\hline
\end{tabular}

respuestas fueron categorizadas según una escala que varió de cero a cuatro puntos, asignando cero el desconocimiento o escaso conocimiento del tópico y 4 al conocimiento total o aplicación de la práctica adecuada (Cuadro 1).

El tamaño de la muestra del estudio se fijó en 400 individuos seleccionados al azar, utilizando como marco de muestreo el censo generado a partir de una encuesta socioeconómica realizada por la alcaldía del municipio Cajigal en la parroquia Yaguaraparo en el año 2009. El tamaño de la muestra correspondió al de una encuesta para la estimación de proporciones, utilizando varianza máxima $(\mathrm{P}=0,5)$, error admisible de 5\% y nivel de confianza de 95\%. (16).

Se trabajó con un Consentimiento Informado acorde a las normas éticas en consideración de la Declaración Universal de Derechos Humanos (17) y la Declaración de Helsinki de la Asociación Médica Mundial adoptada por la Asamblea Médica Mundial de 1964 y sucesivas enmiendas (18).

Las respuestas categorizadas se almacenaron en una base de datos construida en el programa MS Excel 2007. Se tomó como respuesta para cada individuo encuestado el promedio de las respuestas en cada dimensión, de tal manera que cada uno presentó cinco respuestas, una por cada dimensión considerada en el estudio. Se calcularon los estadísticos descriptivos media aritmética, desviación y error estándar, sobre tales respuestas; asimismo, se llevó a cabo el análisis de varianza (ANOVA) y se aplicó la prueba de comparaciones múltiples de Tukey para las diferentes dimensiones considerando las variables intervinientes sexo, grupo etario, grado académico y ocupación. Se utilizó el análisis paramétrico por cuanto el teorema del límite central garantiza la distribución gaussiana de los datos debido al tamaño de muestra utilizado en el estudio (19). Se trabajó al nivel de significación de $5 \%$, por lo cual un efecto se consideró significativo siempre 
Lepidopterismo por $H$. metabus en Yaguaraparo

que $p \leq 0,05$. Los datos se procesaron utilizando el software Statistix 9.0 para Windows.

\section{RESULTADOS}

La muestra estuvo conformada por $65 \%$ (260/400) de individuos de sexo femenino. Adicionalmente, 42,25\% (169/400) de los encuestados tenía edades comprendidas dentro de la adultez joven (20 a 39 años), 34\% (136/400) en la etapa de adultez madura (40 a 59 años) y $21 \%(84 / 400)$ pertenecían a la adultez mayor (60 años en adelante). Por otra parte, 13,75\% (55/400) de los encuestados era analfabeta, 21\% (84/400) tenía formación media diversificada y 21,75\% (87/400) había alcanzado la educación universitaria.

Con respecto a las nociones sobre la patología, 98,5\% (394/400) de los encuestados refirió desconocer el término lepidopterismo y 80\% (320/400) negó conocer el nombre científico Hylesia metabus. Sin embargo, todos los encuestados conocían algo en relación con "estar picado de palometa", así como todos sabían a cuál insecto hacía referencia el nombre "palometa peluda". Igualmente, 98,8\% (395/400) de los participantes describió las características del insecto (llamándolo mariposa) en base a su color marrón o gris, peluda, su vuelo característico y su dimorfismo sexual. También, 79,5\% (318/400) de los encuestados indicó que $H$. metabus podría encontrarse en los manglares, 13,5\% (54/400) relacionó el hábitat del insecto con caños y plantas de guayaba (Psidium guajava), 5,8\% (23/400) relacionó a la mariposa con otros hábitats diferentes del manglar; y solo 1,25\% (5/400) manifestó no conocer el hábitat natural de H. metabus.

Con respecto a la causa de la dermatitis, 98,5\% (394/400) respondió que la urticaria se debía al contacto con el pelo o seta de la mariposa. En cuanto al conocimiento de las formas que causan la urticaria o manifestaciones, solo $33,8 \%$ (135/400) contestó correctamente nombrando a las hembras y a las larvas. Las respuestas indicaron que existe confusión en la comunidad respecto a cuál forma del insecto causa la dermatitis (Cuadro 2).

Los principales síntomas de lepidopterismo mencionados fueron "ronchas y rosetas" 50\% (200/400), "piel gruesa e hinchada" 17\% (68/400) y "picazón" 14\% (56/400). Todos los encuestados consideraron que la "palometa peluda" es un problema para la comunidad, y justificaron esta respuesta al mencionar que esta mariposa es causante de "alergia, hinchazón y picazón" en un 45,3\% (181/400), de "angustia, zozobra y desesperación" según expresó un 23,5\% (94/400) y que "por su culpa, el pueblo debía permanecer a oscuras" dijo un 12,5\% (63/400) (Cuadro 2).

En cuanto a experiencia, 97\% (388/400) de los encuestados indicó que ellos o algún familiar habían sido afectados por la aparición de H. metabus. De estos, 68,8\% (275/400) buscó atención médica, y el 28,25\% (113/400) restante no lo hizo, por preferir el tratamiento casero, la automedicación o porque no concedió importancia a los síntomas. Las causas más frecuentes citadas por los encuestados para acudir al hospital fueron la aparición de alergia con rosetas, piel gruesa y roja (Cuadro 2).

Con respecto al conocimiento de las medidas para evitar el contacto con H. metabus, la acción declarada con mayor frecuencia fue apagar las luces 50,3\% (201/400), y la práctica más frecuente, permanecer en sus casas $14 \%$ (56/400), con las primeras respuestas obtenidas (Cuadro 3).

Respecto a las prácticas, la primera acción realizada al ser afectados por el insecto fue la de rascarse la piel 34,5\% (138/400), como respuesta primaria al prurito; el resto de las respuestas indicó el uso de uno o varios compuestos y productos para mitigar los efectos del lepidopterismo. Solo 8,5\% (34/400) de los encuestados afirmó que su primera acción fue tomar antialérgicos, y $9 \%$ (36/400) declaró no ser afectado por H. metabus (Cuadro 4). 


\section{Herrera-Chaumont et al}

\section{Cuadro 2}

Conocimientos sobre Hylesia metabus y lepidopterismo

\begin{tabular}{|c|c|c|}
\hline 2.1 ¿Quien pica? & Frecuencia & Porcentaje \\
\hline Hembra y gusanos & 135 & 33,75 \\
\hline Macho, hembra y gusanos & 76 & 19,00 \\
\hline Todas las formas & 49 & 12,25 \\
\hline No sabe & 47 & 11,75 \\
\hline Hembra, gusanos y huevos & 39 & 9,75 \\
\hline Hembra solamente & 29 & 7,25 \\
\hline Macho y hembra & 14 & 3,50 \\
\hline Hembra y huevos & 4 & 1,00 \\
\hline Macho solamente & 4 & 1,00 \\
\hline Gusano solamente & 3 & 0,75 \\
\hline TOTAL & 400 & 100 \\
\hline \multicolumn{3}{|l|}{2.2 ¿Por qué es un problema? } \\
\hline Da alergia, hinchazón y picazón & 181 & 45,25 \\
\hline Da angustia, zozobra, deseperación & 94 & 23,50 \\
\hline El pueblo está a oscuras & 63 & 12,50 \\
\hline Contamina el ambiente & 10 & 2,50 \\
\hline El animal bota pelos & 7 & 1,75 \\
\hline Algunos son alérgicos & 7 & 1,75 \\
\hline Paraliza economía, turismo, educación & 6 & 1,50 \\
\hline La gente no puede salir & 5 & 1,25 \\
\hline Le hace daño a los niños & 3 & 0,75 \\
\hline Da incomodidad & 2 & 0,50 \\
\hline Otros & 22 & 5,50 \\
\hline TOTAL & 400 & 100 \\
\hline \multicolumn{3}{|l|}{2.3 ¿Por cuál razón fueron al hospital?* } \\
\hline Alergia (ronchas, hinchazón, piel roja) & 251 & 91,00 \\
\hline Comezón y urticaria & 9 & 3,27 \\
\hline Dificultad para respirar & 4 & 1,45 \\
\hline Ojos hinchados & 3 & 1,09 \\
\hline Piel hinchada & 2 & 0,72 \\
\hline Quemaduras & 2 & 0,72 \\
\hline Nervios atacados & 2 & 0,72 \\
\hline Llagas en la piel & 1 & 0,36 \\
\hline Vómitos & 1 & 0,36 \\
\hline TOTAL & 275 & 100 \\
\hline
\end{tabular}

(*) Estas frecuencias incluyen solo la primera respuesta, en el orden de tres obtenidas

\section{Revista Biomédica}


Lepidopterismo por $H$. metabus en Yaguaraparo

Cuadro 3

Conocimiento sobre las medidas para evitar el contacto con la palometa onocimientos sobre Hylesia metabus y lepidopterismo

\begin{tabular}{lcc}
\hline ¿Cuales medidas conoces? & Frecuencia & Porcentaje \\
\hline $\begin{array}{l}\text { Apagar las luces, bombillos, } \\
\text { estar a oscuras }\end{array}$ & 201 & 50,2 \\
$\begin{array}{l}\text { Encerrarse, no salir de sus } \\
\text { casas }\end{array}$ & 56 & 14,0 \\
$\begin{array}{l}\text { Alejarse de ellas, esquivarlas, } \\
\text { huirles }\end{array}$ & 35 & 8,75 \\
$\begin{array}{l}\text { Ninguna porque igual te } \\
\text { pican }\end{array}$ & 24 & 1,25 \\
Cerrar las puertas & 21 & 5,25 \\
$\begin{array}{l}\text { Desconoce } \\
\text { Otras }\end{array}$ & 16 & 4,00 \\
TOTAL & 47 & 11,75 \\
\hline
\end{tabular}

En cuanto a las medidas empleadas para eliminar H. metabus del ambiente, la respuesta más frecuente fue la fumigación con gasoil o solución jabonosa 68,5\% (274/400) y apagar las luces 16,75\% (67/400) (Cuadro 4).

Con respecto a las medidas para evitar la entrada de la mariposa a sus casas, las acciones declaradas en mayor frecuencia fueron cerrar puertas y ventanas 61\% (244/400), y apagar las luces 32,5\% (130/400), el resto de las medidas incluyeron lavar la casa, matar las mariposas y pintar los bombillos (Cuadro 4).

Se observaron diferencias significativas entre los grupos etarios $(\mathrm{p}=0,0108)$, siendo el grupo de 30 a 39 años el que presentó un mejor grado de conocimiento $(\bar{x}=2,70)$; igualmente para los sexos $(p=0,0483)$, siendo el sexo masculino quien tenía mejor grado de conocimiento $(\bar{x}=2,67)$. Asimismo, se encontraron diferencias significativas para el grado de instrucción ( $p=0,0001)$, siendo las personas con formación universitaria quienes presentaron mejor grado de conocimiento ( $\overline{\mathrm{x}}$ $=2,87$ ). De igual manera el conocimiento respecto a $H$. metabus presentó diferencias significativas según la ocupación de los encuestados $(\mathrm{p}<0,0001)$, obteniéndose que la categoría conformada por

\section{Cuadro 4}

Prácticas. Acciones ante el lepidopterismo y hacia la Hylesia metabus

\begin{tabular}{|c|c|c|}
\hline & Frecuencia & Porcentaje \\
\hline \multicolumn{3}{|c|}{ 4. 1 ¿Qué es lo primero que haces si te ha picado la palometa? } \\
\hline Rascarse la piel & 138 & 34,50 \\
\hline $\begin{array}{l}\text { Echarse alcohol y sus } \\
\text { combinaciones }\end{array}$ & 94 & 23,50 \\
\hline Nada, afirma que no le pica & 36 & 9,00 \\
\hline Tomar antialérgicos & 34 & 8,50 \\
\hline $\begin{array}{l}\text { Echarse mentol o alcanfor, más } \\
\text { alcohol, aceite o talco }\end{array}$ & 32 & 8,00 \\
\hline Bañarse & 18 & 4,50 \\
\hline $\begin{array}{l}\text { Echarse vinagre, kerosén, limón } \\
\text { gasoil }\end{array}$ & 16 & 4,00 \\
\hline Echarse Caladryl ${ }^{\circledR}$ & 14 & 3,50 \\
\hline Usar cremas & 10 & 2,50 \\
\hline Otros & 8 & 2,00 \\
\hline TOTAL & 400 & 100 \\
\hline \multicolumn{3}{|c|}{ 4.2 Medidas para eliminar la palometa del ambiente } \\
\hline $\begin{array}{l}\text { fumigación; gasoil y/o agua con } \\
\text { detergente }\end{array}$ & 274 & 68,50 \\
\hline Apagar las luces, bombillos & 67 & 16,75 \\
\hline Poner trampas de luz, poner focos & 17 & 4,25 \\
\hline quemarlas, hacen hogueras & 10 & 2,50 \\
\hline Insecticida o veneno & 5 & 1,25 \\
\hline No sabe & 11 & 2,75 \\
\hline Otros & 16 & 4,00 \\
\hline TOTAL & 400 & 100 \\
\hline \multicolumn{3}{|c|}{ 4.3 Medidas para evitar la entrada de la palometa a sus casas } \\
\hline Cerrar puertas y ventanas & 244 & 61,00 \\
\hline Apagar las luces & 130 & 32,50 \\
\hline Otros & 26 & 6,50 \\
\hline TOTAL & 400 & 100 \\
\hline
\end{tabular}

profesionales (docentes, enfermeras, ingenieros, administradores, paramédicos, entre otros) presentó mejores respuestas $(\overline{\mathrm{x}}=2,89)$, que el resto de las ocupaciones (Cuadro 5).

Se observaron diferencias significativas en cuanto al conocimiento sobre lepidopterismo según el grupo etario $(\mathrm{p}=0,0044)$, siendo los grupos de edades intermedias de 20 a 29 


\section{Herrera-Chaumont et al}

\section{Cuadro 5}

Grupos de medias de la prueba de comparaciones múltiples de Tukey para las variables intervinientes grupo etario, grado de instrucción y ocupación

\begin{tabular}{|c|c|c|c|c|c|}
\hline & & $\begin{array}{l}\text { Conocimiento } \\
\text { sobre H. metabus }\end{array}$ & $\begin{array}{c}\text { Conocimiento } \\
\text { sobre } \\
\text { lepidopterismo }\end{array}$ & $\begin{array}{c}\text { Conocimientos } \\
\text { y prácticas en } \\
\text { general }\end{array}$ & $\begin{array}{l}\text { Prevención de } \\
\text { lepidopterismo }\end{array}$ \\
\hline & Categorias & \multicolumn{4}{|c|}{ Medidas aritméticas } \\
\hline \multicolumn{6}{|l|}{$\begin{array}{l}\text { Grado de } \\
\text { instrucción }\end{array}$} \\
\hline & Analfabeta & $2,470^{\mathrm{B}}$ & $1,406^{\mathrm{B}}$ & $2,285^{\mathrm{C}}$ & $2,418^{\mathrm{B}}$ \\
\hline & Primaria incompleta & $2,516^{\mathrm{B}}$ & $1,560^{\mathrm{AB}}$ & $2,327^{\mathrm{BC}}$ & $2,371^{\mathrm{B}}$ \\
\hline & Primaria completa & $2,559^{\mathrm{B}}$ & $1,564^{\mathrm{AB}}$ & $2,370^{\mathrm{BC}}$ & $2,408^{\mathrm{B}}$ \\
\hline & Básica incompleta & $2,497^{\text {в }}$ & $1,586^{\mathrm{AB}}$ & $2,322^{\mathrm{BC}}$ & $2,482^{\mathrm{AB}}$ \\
\hline & Básica completa & $2,608^{\mathrm{B}}$ & $1714^{\mathrm{A}}$ & $2,480^{\mathrm{AB}}$ & $2,571^{\mathrm{AB}}$ \\
\hline & Div. incompleta & $2,333^{\mathrm{B}}$ & $1694^{\mathrm{AB}}$ & $2,300^{\mathrm{BC}}$ & $2,444^{\mathrm{AB}}$ \\
\hline & Div. completa & $2,618^{\mathrm{B}}$ & $1,784^{\mathrm{A}}$ & $2,473^{\mathrm{B}}$ & $2,565^{\mathrm{AB}}$ \\
\hline & Universitario & $2,870^{\mathrm{A}}$ & $1,769^{\mathrm{A}}$ & $2,609^{\mathrm{A}}$ & $2,720^{\mathrm{A}}$ \\
\hline \multicolumn{6}{|l|}{ Grupo etario } \\
\hline & 10 a 19 & $2,701^{\mathrm{AB}}$ & $1,575^{\mathrm{AB}}$ & $2,480^{\mathrm{A}}$ & \\
\hline & 20 a 29 & $2,663^{\mathrm{AB}}$ & $1,735^{\mathrm{A}}$ & $2,472^{\mathrm{A}}$ & \\
\hline & 30 a 39 & $2,709^{\mathrm{A}}$ & $1,663^{\mathrm{AB}}$ & $2,483^{\mathrm{A}}$ & \\
\hline & 40 a 49 & $2,592^{\mathrm{AB}}$ & $1,761^{\mathrm{A}}$ & $2,469^{\mathrm{A}}$ & \\
\hline & 50 a 59 & $2,617^{\mathrm{AB}}$ & $1,698^{\mathrm{AB}}$ & $2,456^{\mathrm{A}}$ & \\
\hline & 60 a 69 & $2,619^{\mathrm{AB}}$ & $1,564^{\mathrm{AB}}$ & $2,377^{\mathrm{B}}$ & \\
\hline & 70 a 79 & $2,365^{\mathrm{B}}$ & $1,370^{\mathrm{B}}$ & $2,211^{\mathrm{B}}$ & \\
\hline & 80 y más & $2,387^{\mathrm{AB}}$ & $1,426^{\mathrm{AB}}$ & $2,236^{\mathrm{B}}$ & \\
\hline \multicolumn{6}{|l|}{ Ocupación } \\
\hline & Ama de casa & $2,495^{\text {B }}$ & & & \\
\hline & Estudiante & $2,687^{\mathrm{AB}}$ & & & \\
\hline & Obrero & $2,652^{\mathrm{B}}$ & & & \\
\hline & Comerciante & $2,551^{\mathrm{B}}$ & & & \\
\hline & Técnicos & $2,751^{\mathrm{AB}}$ & & & \\
\hline & Profesionales & $2,897^{\mathrm{A}}$ & & & \\
\hline & Jubilados & $2,714^{\mathrm{AB}}$ & & & \\
\hline
\end{tabular}

Nota: Medias con igual letra no presentan diferencias estadísticamente significativas al 5\%.

años $(\overline{\mathrm{x}}=1,76)$ y de 40 a 49 años $(\overline{\mathrm{x}}=1,73)$, los que mostraron respuestas que sugirieron un mayor nivel de conocimiento. Asimismo, se encontraron diferencias significativas para el grado de instrucción $(p=0,0001)$, siendo los individuos con grado universitario quienes dieron mejores respuestas $(\overline{\mathrm{x}}=1,78)$. No se observaron diferencias significativas para las demás variables intervinientes (Cuadro 5).

Las prácticas de prevención que realiza la población no mostraron diferencias significativas según el grupo etario, sexo u ocupación del

\section{Revista Biomédica}


Lepidopterismo por $H$. metabus en Yaguaraparo

encuestado; solamente con respecto al grado de instrucción se observaron diferencias significativas ( $<<0,0001$ ), siendo los individuos con formación universitaria quienes realizaban las mejores prácticas para la prevención del lepidopterismo $(\overline{\mathrm{x}}=2,72)$ (Cuadro 5). Las prácticas de control de lepidopterismo aplicadas no mostraron diferencias significativas para ninguna de las variables intervinientes consideradas.

Al integrar los aspectos estudiados como conocimientos y prácticas en general, se obtuvieron diferencias significativas para el grupo etario $(p=0,0004)$, presentando un comportamiento similar los grupos comprendidos entre 18 y 59 años. Igualmente, para el grado de instrucción $(p<0,0001)$, corroborando que a mayor grado de instrucción, son mejores los conocimientos y prácticas en general: el grupo con grado universitario presentó las mejores respuestas $(\overline{\mathrm{x}}=2,60)$. Respecto a la ocupación $(p<0,0001)$, los profesionales presentaron mejor grado de conocimientos y prácticas en general $(\overline{\mathrm{x}}=2,48)($ Cuadro 5).

\section{DISCUSIÓN}

Al analizar las respuestas sobre el sitio de cría de esta mariposa, se evidenció que gran parte de los entrevistados tenía buenas nociones de acuerdo al conocimiento formal. La mayoría de los encuestados respondió de manera acertada que $H$. metabus crece en los bosques manglares y otros hospederos tales como plantas de guayaba (Psidium guajava). Con respecto al momento de llegada de la mariposa a las comunidades, gran parte de los encuestados respondió acertadamente que aparecía en la noche y la gran mayoría especificó el horario comprendido entre las 18:00 y 19:00 horas. Casi la totalidad de los encuestados la describió correctamente como una mariposa marrón claro o gris oscuro y además con presencia de pelos o setas, asimismo, los participantes afirmaron que la causa de la urticaria y otras manifestaciones era el pelo o la seta que se desprendía de la mariposa.

Este conjunto de aspectos indica que en general el conocimiento de la población sobre H. metabus es bueno y puede superar el valor promedio, hallazgo similar al de García et al (20), quienes exploraron estas nociones en comunidades del estado Delta Amacuro, Venezuela, observando un buen nivel de conocimiento de los habitantes sobre aspectos de la biología de la mariposa. Sin embargo, aunque las nociones en la parroquia Yaguaraparo sobre $H$. metabus califican como buenas, no son óptimas, ya que al preguntar “¿Quién pica?” la población señaló de manera dispersa las distintas formas evolutivas o sexuales que al tener contacto directo o indirecto con la persona, la afectaban. La opción correcta, hembras y larvas, fue mencionada por menos de la mitad de la muestra, y un grupo menor de personas no sabía o pensaba que todas las formas "picaban", lo cual indica dónde se requiere ajustar este conocimiento.

La mayoría de los participantes desconocía el nombre científico Hylesia metabus, pero la reconocía bien como "palometa peluda". Nótese que la denominación que le dan es de mariposa, no de polilla, aunque desde el punto de vista morfológico y etológico debería llamársele polilla. Igualmente, la literatura disponible le llama mariposa, lo cual favorece la denominación general para lepidópteros.

Con respecto a la percepción de $H$. metabus como problema de salud, la totalidad de los encuestados la catalogó como generadora de problemas de salud y alteración de la calidad y estilo de vida. Sin embargo, al responder una pregunta previa sobre los principales problemas de salud en su comunidad, solo pocos participantes nombraron la aparición de esta mariposa como tal. Situación similar describieron en la misma parroquia Sojo-Milano et al (21), quienes señalaron la baja percepción de los habitantes respecto a ubicar $H$. metabus entre sus principales problemas de salud. Posiblemente la fuerza 


\section{Herrera-Chaumont et al}

de esta apreciación varía con muchos factores siendo la estacionalidad uno de ellos, es decir, considerarla o no un problema cambia según la presencia o cercanía de un ciclo de aparición de la mariposa y por ende, de una temporada donde el lepidopterismo deviene como algo visible en la comunidad. Otro elemento posible es que ante la importancia relativa o el impacto de otros problemas de salud, para la población general, el lepidopterismo califique más como una molestia que como un problema de salud propiamente dicho. Para el momento de la encuesta que aquí analizamos se habían cumplido cuatro años sin la presencia de $H$. metabus en la parroquia.

Gran parte de los participantes no sabía que lepidopterismo es el nombre de la entidad clínica que sufrían por el contacto con la mariposa o sus setas urticantes. En cambio, se reconocían como "picados de palometa". Con respecto a los síntomas que se generaban con dicho contacto, casi la totalidad de los entrevistados enfocó sus respuestas solo en signos y síntomas de la dermatitis urticante (ronchas, rosetas, piel roja e hinchada, picazón), mencionando poco el compromiso de otros sistemas. Otros trabajos describen hallazgos similares, donde la población asoció el lepidopterismo solamente con el prurito y la erupción de la dermatitis urticante, sin mencionar otras manifestaciones $(9,20)$.

Al indagar sobre el conocimiento de las medidas para evitar el contacto con la palometa, la mayoría demostró conocer al menos una acción. Según el conocimiento formal hay varias medidas relacionadas con la vestimenta, el apagado de las luces, el cuidado de las áreas de la vivienda y de la vegetación que contribuyen a la disminución de la exposición y contacto con la mariposa (11). La medida más frecuentemente señalada por los encuestados en Yaguaraparo fue el apagado de las luces.

En líneas generales, las prácticas descritas por las personas para evitar el contacto directo o indirecto con la mariposa calificaron con calidad de regular a buena. Casi todos los participantes mencionaron al menos una acción, y las principales respuestas para evitar el lepidopterismo fueron apagar las luces, encerrarse en la casa y cerrar puertas y ventanas, indicando con ello que se desestiman otras medidas, tales como el uso de barreras físicas, (telas metálicas) y de ordenamiento del medio (limpieza de áreas externas de las viviendas), lo cual introduce el tema de costos y de prácticas de saneamiento ambiental.

Un pequeño número de encuestados refirió no realizar algún tipo de actividad para prevenir el contacto con $H$. metabus porque pensaban que era inevitable, el número de individuos con esta creencia fue mayor en otros estudios (20). En todo caso, aunque sea cuantitativamente menor, debe prestarse atención a esta actitud, ya que es un elemento a tomar en cuenta en las estrategias de comunicación en general y de comunicación de riesgos en particular pues aceptar que algo es inevitable, favorece la desmotivación, la inacción y afecta la autogestión. Aunque fue la categoría que obtuvo el mejor puntaje, tampoco llegó a ser óptimo, por la combinación de acciones incorrectas y aconsejables, con efecto neutralizante. Un ejemplo importante fue que más de un tercio de los entrevistados se "rascaban" al momento de sufrir lepidopterismo, acción que está contraindicada, ya que el pelo, seta o tricoma, se introduce más en la piel, empeorando el cuadro clínico. Otra medida mencionada fue la ingesta de antialérgicos. Estas dos últimas prácticas fueron predominantes entre los encuestados y solo un pequeño porcentaje mencionó que prefería ir al hospital en busca de atención médica.

Cuando se indagó la frecuencia que buscaba atención médica, una alta proporción de los afectados declaró hacerlo ante las manifestaciones de dermatitis. Otros autores han reportado frecuencias menores, lo que refleja un mayor número de personas que aun estando afectadas, optan por no acudir a los centros de salud. Más de la mitad y casi la totalidad de los encuestados afectados con cuadros clínicos

\section{Revista Biomédica}


Lepidopterismo por $H$. metabus en Yaguaraparo

leves no buscaron atención médica debido a que se atendían con tratamiento casero y/o automedicación $(20,22)$. Impresiona la práctica de la automedicación, aun estando contraindicada por las autoridades de salud. Con respecto al tratamiento casero, se evidenció que existe una variedad de opciones, en donde los encuestados prefirieron el uso de alcohol isopropílico solo o combinado con mentol o alcanfor, además de geles y cremas a base de mentol.

Con respecto a las acciones antiprurito, se recomienda el uso de agentes antipruríticos tópicos, tales como lociones de Calaminol o Calamina, ya que genera sensación de frescura y alivio en la piel irritada. $(23,24)$. Además, se recomienda aplicarse compresas de agua fría y solución de agua más vinagre blanco comestible (ácido acético), porque la proteasa o "peludasa" se inactiva a $\mathrm{pH}$ ácido. La aplicación de una solución de cristales de mentol disueltos en alcohol isopropílico, actúa como calmante. El baño no debe acompañarse con jabones corrientes (su pH entre 8 y 9 aumenta la actividad irritante de la peludasa), y se debe evitar el rascado excesivo de las lesiones, ya que los pelos urticantes se incrustan más (11).

Las personas con grado de instrucción universitario y ocupación en la categoría de profesionales presentaron las mejores respuestas de acuerdo al conocimiento formal. Esto sugiere que el conocimiento y el acceso a la información favorecerían prácticas de control y prevención positivas e incluso que estos grupos poblacionales podrían apoyar y multiplicar las iniciativas institucionales en promoción de la salud. Algo para ser verificado en su contexto, ya que otros autores han señalado que el conocimiento de las medidas preventivas y de control no es garantía del tiempo y el interés que las poblaciones dediquen a ellas $(21,25)$, lo cual indica que es factible, localmente, describir distintos niveles de disociación/integración entre percepciones, conocimientos y prácticas (26).

¿Cómo contribuyen estos resultados a reducir el problema eventual de lepidopterismo por H. metabus? Creemos que someter al contexto el uso o el procesamiento del conocimiento formal es una buena forma de aproximar soluciones. Saber que el sitio de cría de $H$. metabus corresponde al bosque manglar y fuera de él, a plantas tan ubicuas como el guayabo (Psidium guajava), posa el problema en el entorno de las comunidades y al alcance de sus decisiones. Sus hábitos se modifican con los horarios nocturnos de llegada de este insecto, distinto de otros y cuyas setas o pelos, son reconocidos como la causa de la urticaria. Saber identificar a la hembra y a las larvas, que son las formas que se desprenden de estas setas, junto a la capacidad para diagnosticar las manifestaciones del lepidopterismo, (para el cual se tiene un nombre local) e identificar cuáles prácticas evitan el contacto o favorecen su control, elabora un continuo de suma importancia tanto para los habitantes locales como para los conductores del programa.

Los resultados indican la necesidad de ampliar, de diversificar la información sobre el valor protector de la vestimenta y la limpieza alrededor de las viviendas, además del apagado de las luces y medidas de protección individual. Evitar frotarse o rascarse la piel es un mensaje sencillo de extrema importancia, para reducir el uso de medicamentos y evitar la automedicación como una opción.

Hemos presentado evidencias sobre el perfil de las nociones de la población de Yaguaraparo respecto al control y la prevención del lepidopterismo a partir de experiencias previas marcadas por las apariciones de $H$. metabus. Este conjunto de hallazgos representa insumos para basar mejores estrategias de comunicación, información y educación sobre este tema específico. Se puede con ello reforzar el marco de protección-empoderamiento que materializa las políticas de salud e integra a la comunidad.

Como problema de salud pública, 


\section{Herrera-Chaumont et al}

para el ámbito programático es una necesidad conocer lo que las personas piensan de los problemas de salud, porque esta es la base de sus acciones para aportar a su propio bienestar. La experiencia en Venezuela, señala que, la infestación por $H$. metabus y el lepidopterismo crean escenarios complejos, de alta exigencia gerencial, técnica-operacional y científica para resolver conflictos y tensiones entre los diferentes actores sociales, donde el mayor desafío está en reducir la vulnerabilidad de las comunidades. Es necesario, por tanto, garantizar la protección y el acceso a la salud. Una manera de lograrlo es enfatizar las medidas de prevención a todo nivel y de promoción de una salud integral, para mitigar, en lo posible, los efectos desfavorables del lepidopterismo y su potencial para afectar la calidad de vida y el desarrollo integral de las personas. Creemos, que decisiones políticas de salud adecuadas y oportunas y el trabajo con mensajes sencillos y efectivos, pueden dar grandes resultados, cuando se basan en evidencias como las que aquí se presentan.

\section{AGRADECIMIENTOS}

Al Dr. Nunzio Pizzo, a la Ing. Carmen Rosa Méndez, al Dr. Néstor Rubio y a los habitantes de la parroquia Yaguaraparo por su amable colaboración.

\section{REFERENCIAS}

1. Dinehart SM, Archer ME, Wolf JE, McGavran MH, Reitz C, Smith EB, et al. Caripito itch: Dermatitis from contact with Hylesia moths. J Am Acad Dermatol. 1985 Nov; 13(5 Pt 1): 743-7.

2. Diaz JH. The evolving global epidemiology, syndromic classification, management, and prevention of caterpillar envenoming. Am J Trop Med Hyg. 2005 Mar; 72(3): 347-57.

3. Jourdain F, Girod R, Vassal JM, Chandre F, Lagneau C, Fouque F, et al. The moth Hylesia metabus and French Guiana lepidopterism: centenary of a public health concern. Parasite. 2012 May; 19(2): 117-28.

4. Benaim-Pinto C. Reacciones cutáneas indeseables producidas por insectos. Derm Venezol. 2002; 40(4): 90-3.

5. Fornés L \& Hernández JV. Reseña histórica e incidencia en la salud pública de Hylesia metabus (Cramer) (Lepidóptera: Saturniidae) en Venezuela. Entomotropica. 2001 Ago; 16(2): 137-41.

6. Rodríguez-Morales AJ, Arria M, Rojas-Mirabal J, Borges E, Benitez JA, Herrera M, et al. Short Report: Lepidopterism due to exposure to the moth Hylesia metabus in northeastern Venezuela. Am J Trop Med Hyg. 2005 Nov; 73(5): 991-3.

7. Hernández JV, Osborn F, Herrera M. Rasgos de Hylesia metabus (Cramer, [1775]). In: Hernández JV, Osborn F, Conde J. Estudio multidisciplinario de la palometa peluda Hylesia metabus. Caracas: Ediciones IVIC; 2012. P. 15-24.

8. Bourée P, Bisaro F, Joubert M. Une eruption prurigineuse passagère: la papillonite guyanaise. Revue Francophone des Laboratories. 2014 Juin; 2014(463): 79-82.

9. Rodríguez A, Herrera M, Rojas J, Arria M, Maldonado A, Rubios N, et al. Estudio epidemiológico preliminar del lepidopterismo por Hylesia metabus en el municipio Cajigal, estado Sucre. Act Cient Est. 2003 Oct-Dic; 1(4): 117-27.

10. Toro J, Rojas J, Pizzo NN, Polanco G, Escalona H, Escalona E, et al. Monitoreo de adultos de Hylesia metabus (Palometa Peluda), en el Golfo de Paria, estados Sucre, Monagas y Delta Amacuro. Periodo 2005-2006. En: Resúmenes del XX Congreso Venezolano de Entomología. San Cristóbal, Venezuela, 22 al 26 de Julio de 2007. P. 134.

11. Red de Sociedades Científicas Médicas Venezolanas. Alerta Epidemiológica $\mathrm{N}^{\circ}$ 200. Lepidopterismo por Hylesia metabus (Cramer), palometa peluda. Un problema de salud pública en Venezuela. 2011. http://www.rscmv.org.ve/pdf/ALERTA_200.pdf. [Consultado 04 de abril de 2015].

12. Herrera M \& Osborn F. Plagas agrícolas de Venezuela. Palometa peluda. Hylesia metabus. 2015. http://plagas.miza-ucv.org.ve/index.php/plagas/ficha detalle/457/448. [Consultado 04 de abril de 2015].

13. Google Maps. Parroquia Yaguaraparo. Municipio Cajigal. Estado Sucre. Venezuela. 2015. https://goo. gl/maps/sKToygxJLq32 [Consultado 20 de Octubre de 2015].

14. Instituto Nacional de Estadística. Censo 2011. Parroquia Yaguaraparo. Municipio Cajigal. Estado Sucre. Resultados básicos. Indicadores demográficos. http://www.redatam.ine.gob.ve/Censo2011/index. html. [Consultado 04 de abril de 2015].

15. Delgado L, Gamboa L, León N. Aspectos geográficos relacionados con un problema de salud pública: la malaria en el estado Sucre. Terra. 2000; 16(25): 81-97.

16. Lohr SL. Muestreo: Diseño y análisis. México:

\section{Revista Biomédica}


Lepidopterismo por $H$. metabus en Yaguaraparo

Ediciones Paraninfo; 2000.

17. Naciones Unidas. Declaración Universal de Derechos Humanos. http://www.un.org/es/documents/udhr/. [Consultado 04 de abril de 2015].

18. Asociación Médica Mundial. Declaración de Helsinki. Principios éticos para las investigaciones médicas en seres humanos. 2015. http://www.wma. net/es/30publications/10policies/b3/. [Consultado 04 de abril de 2015].

19. Canavos GC. Probabilidad y estadística: aplicaciones y métodos. México: McGraw-Hill-Interamericana de México, S.A. de C.V.; 1988.

20. García B, Alvarado G, López-Aguilar R. Conocimientos y prácticas sobre Hylesia metabus (Cramer, 1775) y lepidopterismo en la población de Capure, estado Delta Amacuro (Julio-Agosto 2005). Bol Mal Salud Amb. 2009 Ago-Dic; 49(2): 293-301.

21. Sojo-Milano M, Cáceres JL, Sojo-Milano E, Rondón L, González C, Rubio N. Conocimientos, prácticas y percepciones sobre malaria en la parroquia Yaguaraparo, estado Sucre, Venezuela, 2004. Bol Mal Salud Amb. 2008 Ene-Jul; 48(1): 61-71.

22. Alvarado G. Caracterización clínico-epidemiológica del lepidopterismo por Hylesia metabus (Cramer,
1775) en Capure, municipio Pedernales, estado Delta Amacuro. Tesis para obtener el Título de Magister Scientiarum en Epidemiología. Instituto de Altos Estudios “Dr. Arnoldo Gabaldón”. Maracay. Venezuela. 2005.

23. Paniz-Mondolfini AE, Pérez-Álvarez AM, Lundberg U, Fornés L, Reyes-Jaimes O, Hérnandez-Pérez $\mathbf{M}$, et al. Cutaneous lepidopterism: dermatitis from contact with moths of Hylesia metabus (Cramer 1775) (Lepidoptera: Saturniidae), the causative agent of Caripito itch. Int J Dermatol. 2011 May; 50(5): 535-41.

24. PR Vademécum Venezuela. Calamina. 2015. http:// ve.prvademecum.com/producto.php?producto $=3111$. [Consultado 20 de Octubre de 2015].

25. Pineda F \& Agudelo C. Percepciones, actitudes y prácticas en malaria en el Amazonas colombiano. Rev Salud Pública. 2005 Nov; 7(3): 339-48.

26. Sojo-Milano M. Prevalencia y factores asociados a infección malárica en la parroquia Yaguaraparo, Sucre, Venezuela, 2004-2005. Tesis para obtener el título de Doctor en Ciencias de la Salud. Especialidad Epidemiología. Instituto de Medicina Tropical Pedro Kourí. La Habana. Cuba. 2011. 